I NRA Prod. Anim., 2001, 14 (5), 323-335
Y. CHILLIARD, A. FERLAY, M. DOREAU

I NRA, Unité de Recherches sur les Herbi vores, Thei $x, 63122$ St-GenèsChampanelle

Courrie : chi Iliar@clermont.inra.fr

\section{Contrôle de la qualité nutritionnelle des matières grasses du lait par l'alimentation des vaches laitières : acides gras trans, polyinsaturés, acide linoléique conjugué}

La matière grasse du lait et des produits laitiers est souvent mal considérée par le consommateur. Les recherches conduites depuis quelques années montrent pourtant que les acides gras saturés qui entrent dans la composition du lait n'augmentent pas tous le risque cardiovasculaire. Certains acides gras peuvent même avoir un effet bénéfique sur la santé humaine. Moduler les rations des vaches laitières permet de mieux maîtriser les proportions des différents acides gras dans leur lait afin de répondre aux objectifs nutritionnels pour l'homme.

\section{Résumé}

Après un rappel des voies métaboliques et des flux de nutriments qui concourent à la lipogenèse mammaire, cet article est consacré aux principaux effets de l'alimentation sur la composition en acides gras (AG) du lait de ruminant, en particulier les AG polyinsaturés, les AG monoinsaturés trans et l'acide linoléique conjugué ( $C L A)$. Les principaux facteurs alimentaires étudiés sont la nature des fourrages (dont l'herbe pâturée), le suif, protégé ou non, et la supplémentation des rations avec des huiles végétales ou marines (poisson ou algues), protégées ou non. La supplémentation en suif augmente la sécrétion d'acide oléique. La teneur du lait en C 18:2 est comprise entre 2 et $3 \%$ avec les rations non supplémentées en lipides, elle n'est augmentée que de 1,5 point lors d'un apport de graines ou d'huiles riches en C18:2, en raison d'une hydrogénation ruminale poussée. Les rations à base d'herbe (riches en $C$ 18:3) augmentent légèrement ( 0,5 à 1 point) la proportion de $C 18: 3$ dans le lait par rapport aux rations à base d'ensilage de maïs. La graine de lin permet un accroissement d'environ 0,3 point. La teneur en C 20:5 (EPA) et en C22:6 (DHA) est accrue jusqu'à $0,5 \%$ des AG totaux par l'addition d'huiles marines à la ration. Le taux butyreux du lait peut être fortement diminué par les régimes pauvres en fibres et riches en céréales et/ou par l'administration d'huiles marines ou végétales riches en AG insaturés. Les C 18:1 trans, notamment les C 18:1 trans 11 et trans 10, mais aussi le trans 10, cis 12 CLA, pourraient jouer un rôle dans cette diminution en inhibant la lipogenèse mammaire. Les régimes alimentaires augmentant les proportions des CLA et des C 18:1 trans dans le lait sont ceux qui apportent des précurseurs lipidiques pour la formation de ces AG, ou qui modifient l'activité microbienne associée à I'hydrogénation ruminale des AG polyinsaturés ou l'activité de la désaturase mammaire. L'influence de l'alimentation sur les différents isomères du C 18:1 et du CLA du lait a été peu étudiée.
Les produits laitiers fournissent de 15 à $25 \%$ des matières grasses consommées par I'Homme, et 25 à $35 \%$ des graisses saturées. Pour cette raison, ils ont parfois été la cible de critiques de la part de diététiciens. Mais les connaissances sur l'impact des acides gras (AG) alimentaires sur la santé humaine ont considérablement évolué depuis quelques années (voir par exemple Williams 2000). Le caractère réputé néfaste des AG saturés a été nuancé, tant au plan quantitatif que qualitatif : ainsi l'acide stéarique n'a pas d'effet athérogène. L'effet délétère de certains $A G$ monoinsaturés trans est parfois mentionné, mais il n'a pas été prouvé que l'isomère principal présent dans le lait, le trans vaccénique (C18:1 11-trans) soit associé au risque coronarien. L'intérêt d'un accroissement du rapport n-3/n6 pour les AG polyinsaturés (AGPI) a été démontré. Enfin et surtout, on connaît mieux les propriétés de l'acide linoléique conjugué ou CLA, dont le principal isomère, l'acide ruménique (C18:2 9-cis, 11-trans), présente des propriétés intéressantes, démontrées sur modèles animaux, notamment pour la prévention de certains cancers.

Ces nouveaux éléments permettent de considérer d'un œil neuf l'intérêt de moduler la composition en AG du lait, dans un sens 
bénéfique pour la santé humaine, et de nombreuses recherches leur ont été consacrées depuis 4 à 5 ans. Un moyen naturel et économique d'y parvenir rapidement est de jouer sur la nature de l'alimentation des vaches. Ceci pourra conduire à terme à une segmentation du marché du lait dès sa livraison aux laiteries, avec un bénéfice potentiel pour l'éleveur, et non plus exclusivement grâce aux apports de la technologie de transformation.

L'un des principaux moyens de modulation de la composition des AG du lait est l'apport de supplements lipidiques dans la ration. Cette pratique a des conséquences maintenant bien connues sur la production et les teneurs en matières grasses et en protéines du lait : tendance à l'accroissement de production, diminution faible mais quasi systématique du taux protéique, variation limitée du taux butyreux à l'exception des huiles de poisson, qui entraînent une forte baisse, et des lipides protégés par encapsulation, qui l'accroissent fortement (tableau 1). Dans ce texte, on examinera successivement l'impact des régimes alimentaires des vaches sur les grandes classes d'AG : saturés et monoinsaturés cis, polyinsaturés, et enfin CLA et monoinsaturés trans, ces deux dernières catégories étant souvent fortement corrélées.

\section{1 / Voies métaboliques et flux de nutriments impli- qués dans la synthèse des lipides du lait}

\section{1 / Lipogenèse mammaire}

Les AG du lait ont une double origine (figure 1 ) : ils sont prélevés dans le plasma ( $60 \%$ des AG sécrétés dans le lait), ou ils sont synthétisés de novo dans la glande mammaire ( $40 \%)$ à partir de l'acétate et du $\beta$-hydroxybutyrate (contribuant à environ $15 \%$ des AG néosynthétisés). La principale voie métabolique implique deux enzymes clefs : I'acétylCOA carboxylase (ACC) et l'acide gras synthétase (AGS). La première enzyme catalyse la formation de malonyl-CoA à partir de l'acétate et la seconde catalyse la condensation du
malonyl-CoA avec l'acétyl-CoA, ou le butyrylCoA, produits respectivement à partir du métabolisme de l'acétate et du $\beta$-hydroxybutyrate. L'AGS condense entre elles les unités à 2 carbones jusqu'à l'obtention de chaînes à 14 et surtout a 16 atomes de carbone, qui sont ensuite libérées du complexe enzymatique par une de ses enzymes constitutives (thioestérase I). A l'inverse des rongeurs, I'AGS de la glande mammaire des ruminants peut interrompre le processus de condensation plus précocement, avec la possibilité de libérer des chaînons acyl de 4 à 12 atomes de carbone. Les facteurs cellulaires et moléculaires régulant la formation des AG à chaînes courte et moyenne, à l'intérieur d'une même espèce et entre espèces au sein des ruminants, n'ont cependant pas été identifiés.

Les AG préformés sont véhiculés dans le plasma sous forme d'AG non estérifés (AGNE), ou de lipoprotéines riches en triglycérides (chylomicrons ou lipoprotéines de très basse densité). Le prélèvement des triglycérides par la glande mammaire est permis par la lipoprotéine lipase (LPL). La quantité de TG prélevés est généralement bien corrélée à leur concentration plasmatique, excepté pour des valeurs élevées (au-delà de $0,4 \mathrm{mM}$ ) pour lesquelles l'activité de la LPL devient limitante.

La glande mammaire ne peut pas convertir le C16:0 en C18:0 par élongation de la chaîne carbonée. En outre, les cellules mammaires sécrétrices entièrement différenciées présentent une forte activité de la delta-9 désaturase, qui convertit l'acide stéarique en acide oléique (C18:1 9-cis). Environ 40 \% de l'acide stéarique prélevé par la glande mammaire est désaturé, contribuant ainsi à plus de $50 \%$ de la quantité d'acide oléique sécrétée dans le lait. De plus, l'acide trans-vaccénique (C18:1 11-trans) d'origine ruminale peut être désaturé pour former l'acide ruménique (C18:2 9-cis, 11trans), principal isomère du CLA dans le lait (Griinari et al 2000a ; figure 2). D'autres isomères mineurs du CLA sont probablement synthétisés à partir d'autres isomères du C18:1 trans (Griinari et al 2000b). Une activité élevée de désaturation pourrait expliquer que le rapport CLA (produit)/C18:1 trans (précurseur) dans le lait soit assez peu variable.

Tableau 1. Effets de la supplémentation lipidique sur la production et la composition du lait de vache (effets exprimés par différence avec le lot témoin, d'après Chilliard et al 2001).

\begin{tabular}{|l|c|c|c|c|c|c|}
\hline Lipides alimentaires & $\mathrm{n}^{(1)}$ & $\begin{array}{c}\text { Quantité de lipides } \\
\text { alimentaires } \\
(\mathrm{g} / \mathrm{j})\end{array}$ & $\begin{array}{c}\text { Production } \\
\text { laitière } \\
(\mathrm{kg} / \mathrm{j})\end{array}$ & $\begin{array}{c}\text { Taux } \\
\text { protéique } \\
(\mathrm{g} / \mathrm{kg})\end{array}$ & $\begin{array}{c}\text { Taux } \\
\text { butyreux } \\
(\mathrm{g} / \mathrm{kg})\end{array}$ & $\begin{array}{c}\text { Production de } \\
\text { matières grasses } \\
(\mathrm{g} / \mathrm{j})\end{array}$ \\
\hline Matières grasses animales (MGA) & 22 & 688 & $+0,5$ & $-0,6^{* *}$ & $-1,4$ & -18 \\
MGA encapsulées & 26 & 941 & $+1,0^{*}$ & $-1,8^{* *}$ & $+4,0^{* *}$ & $+143^{* *}$ \\
Acides gras saturés & 10 & 644 & $+1,7^{* *}$ & $-0,6^{*}$ & $+0,5$ & $+58^{*}$ \\
Savons de Ca d'huile de palme & 29 & 593 & $+0,9^{* *}$ & $-1,2^{* *}$ & $+0,4$ & $+47^{* *}$ \\
Huiles végétales & 8 & 573 & $-0,6$ & $-0,9$ & $-2,8^{*}$ & $-74^{*}$ \\
Graines oléagineuses & 34 & 538 & $+0,3$ & $-0,4^{* *}$ & $-0,9^{*}$ & -18 \\
Huiles végétales encapsulées & 26 & 693 & 0,0 & $-0,8$ & $+6,4^{* *}$ & $+120^{* *}$ \\
Huiles marines & 27 & 305 & $+0,2$ & $-1,2^{* *}$ & $-9,1^{* *}$ & $-208^{* *}$ \\
\hline
\end{tabular}

\footnotetext{
(1) Nombre de lots supplémentés.
}

* ou ** : écart significativement différent de zéro $(P<0,05$ ou $P<0,01)$. 
Figure 1. Synthèse et sécrétion des lipides du lait chez les ruminants.

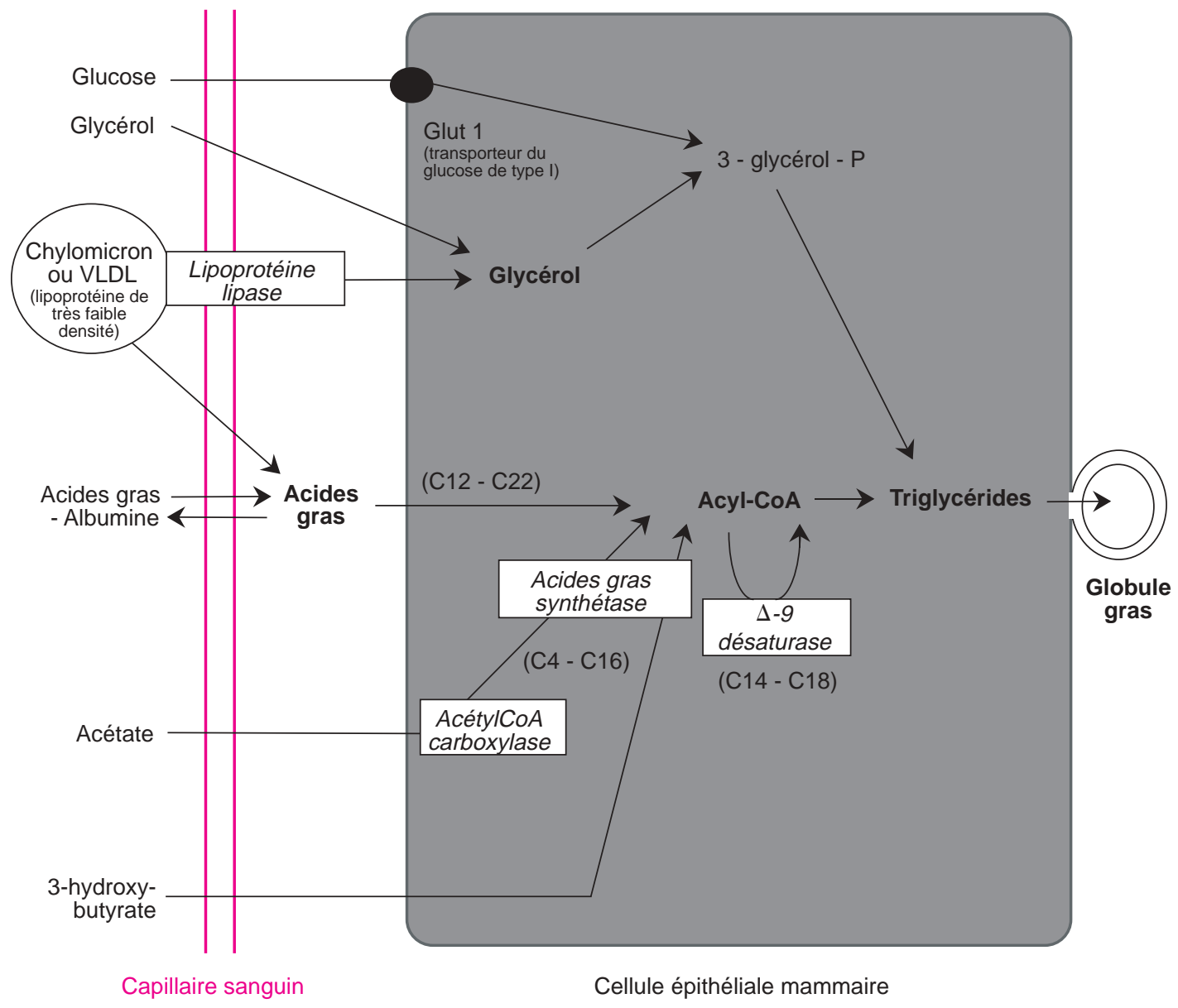

Figure 2. Principales voies de synthèse des acides gras trans et du CLA du lait.

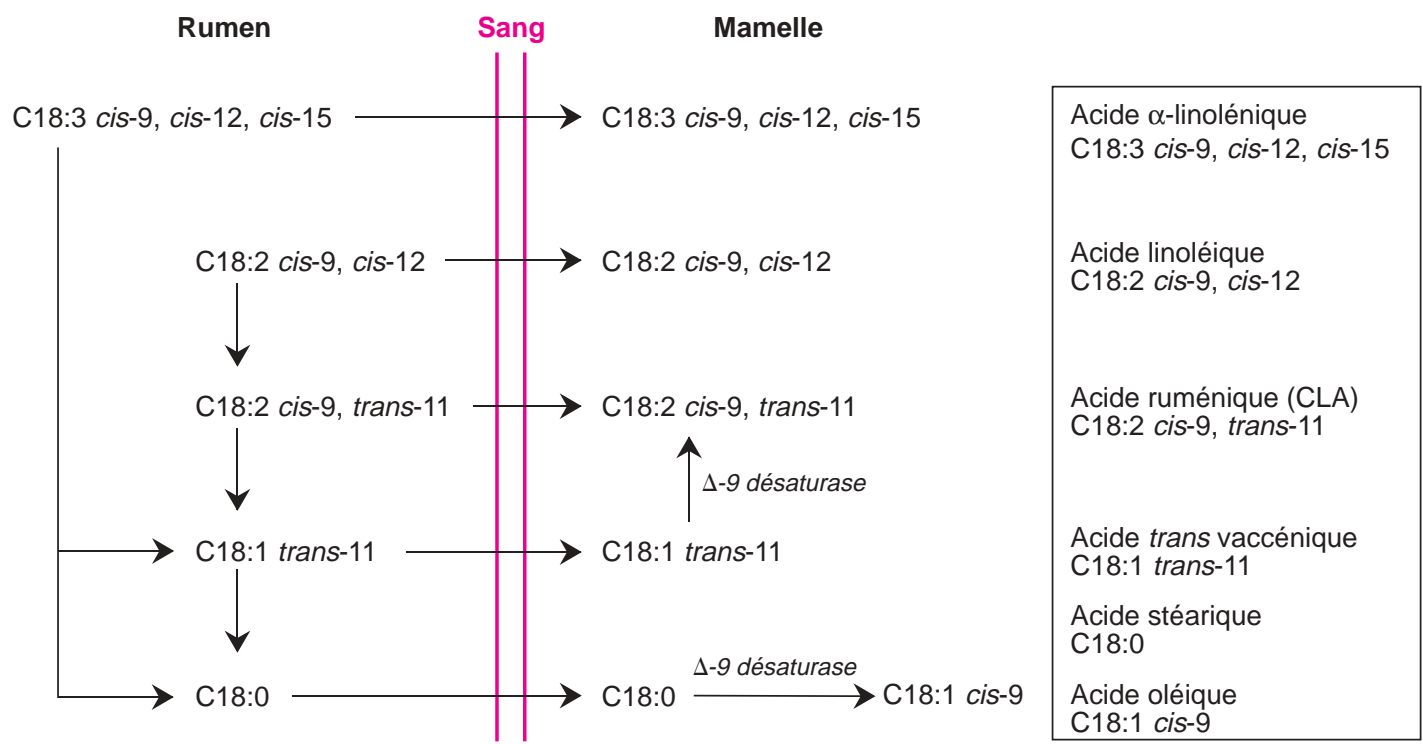

Griinari et Bauman (1999) suggèrent qu'environ $30 \%$ du C18:1 11-trans prélevé par la mamelle soit désaturé en acide ruménique. L'activité de la delta-9 désaturase peut être inhibée par les AGPI et par l'acide cyclopropénoïque des graines de coton.

Ces différentes voies métaboliques (synthèse de novo, prélèvement plasmatique et désaturation) contribuent à la constitution d'un pool d'AG utilisables pour la formation de tri- glycérides (97 à $98 \%$ des lipides totaux du lait) par estérification sur le glycérol. La distribution asymétrique des AG sur la molécule de glycérol influence les propriétés physiques de la matière grasse du lait. Cette particularité s'ajoute aux autres caractéristiques du métabolisme mammaire (synthèse d'AG à courtes chaînes, désaturation des $A G$ à longues chaînes) pour diminuer le point de fusion des matières grasses du lait, ce qui tempère les effets des hydrogénations ruminales. 


\section{2 / Les transformations des lipides dans le rumen}

Le rumen est le lieu d'un métabolisme des lipides de forte intensité, lié à l'activité microbienne. La lipolyse des glycolipides, phospholipides et triglycérides du régime est très rapide et presque complète, et les AG qui en sont issus sont fortement hydrogénés. Les principaux mécanismes biochimiques ont été détaillés par Harfoot et Hazlewood (1988). Ces auteurs ont en particulier décrit les voies biochimiques d'hydrogénation de l'acide linoléique et de l'acide linolénique. Ainsi l'acide linoléique (C18:2 9ds, 12-cis) est isomérisé en acide ruménique (C18:2 9-cis, 11-trans), puis ce dernier est hydrogéné en acide trans-vaccénique (C18:1 11-trans) et enfin en acide stéarique C18:0. L'acide linolénique donne un nombre plus important d'intermédiaires, dont l'acide transvaccénique, mais la production d'acide ruménique n'a pour l'instant pas été montrée (figure 2). En fait, les voies biochimiques sont probablement multiples et beaucoup plus complexes. Dans le milieu ruminal, on ne trouve pas moins de 12 isomères monoinsaturés trans a 18 carbones (Keeney 1970), et de nombreux isomères du CLA ont été signalés.

Le terme CLA (Conjugated Linoleic Acid) regroupe en effet un ensemble d'isomères géométriques et de position (ayant tous des doubles liaisons conjuguées) de l'acide linoléique. La réduction des C18:1 trans est généralement une étape limitante pour I'hydrogénation complète des C18 insaturés, induisant une accumulation ruminale des C18:1 trans, mais rarement celle de CLA, comme suggéré par le faible rapport CLA/C18:1 trans (1/40) (Griinari et Bauman 1999). Une partie du CLA provenant de l'hydrogénation ruminale des AGPI est toutefois absorbée au niveau intestinal, prélevée par la glande mammaire et sécrétée dans le lait. Cependant, la synthèse d'acide ruménique se ferait principalement (pour plus de $75 \%$ ) dans la mamelle (et peut être dans d'autres organes comme le foie), mais pour cela il est nécessaire qu'une quantité suffisante d'acide trans-vaccénique ait été formée dans le rumen.

Le bilan quantitatif et qualitatif du métabolisme ruminal a été précisé par Doreau et Ferlay (1994). L'hydrogénation dans le rumen, généralement définie comme le pourcentage de disparition des acides linoléique et linolénique entre la bouche et le duodénum, est en moyenne de 80 et $92 \%$ pour ces deux AG. L'hydrogénation plus faible de l'acide linoléique peut s'expliquer par une incorporation partielle dans les microbes, mais peutêtre aussi par le fait que les voies biochimiques sont différentes. Le pourcentage d'hydrogénation ne semble pas dépendre de la nature ou de la quantité des AG ingérés, mais plutôt du pourcentage de concentré dans la ration. Quand celui-ci est supérieur à $70 \%$, I'hydrogénation n'est en moyenne que de 50 et $65 \%$ pour les acides linoléique et linolénique. Ce phénomène est relié à une chute du $\mathrm{pH}$ ruminal, qui limite non seulement l'hydrogénation, mais aussi en premier lieu la lipolyse. L'effet de la quantité d'AGPI ingérés, s'il ne INRA Producti ons Ani males, décembe 2001 limite pas la phase initiale de l'hydrogénation, conduit à un accroissement de composés intermédiaires, CLA et AG monoinsaturés trans, au détriment de l'acide stéarique (Noble et al 1974).

\section{3 / Métabolisme du tissu adipeux}

La glande mammaire utilise les AGNE plasmatiques libérés par le tissu adipeux comme source d'AG à longue chaîne pour la synthèse des lipides du lait. Les AG stockés en TG dans les tissus adipeux de ruminant sont principalement le C16:0, le C18:0, le C18:1 9-cis et dans une moindre mesure le C14:0, le C16:1 9-cis, le C17:0, le C18:1 11-trans et des AG mineurs avec de grandes variations entre sites anatomiques. La composition en AG des tissus adipeux dépend aussi des conditions alimentaires. La disponibilité en AGNE plasmatiques pour la glande mammaire est fortement corrélée à la lipomobilisation, qui intervient en début de lactation et/ou quand le bilan énergétique est négatif. La quantité et la nature des lipides alimentaires influencent aussi la mobilisation des réserves corporelles. La composition en AG des AGNE plasmatiques dépend en outre du passé nutritionnel de l'animal, ainsi que de l'ordre suivant lequel sont mobilisés les différents sites de tissus adipeux, et les différents AG à l'intérieur d'un même site.

\section{2 / Maîtrise par l'alimentation de la composition en acides gras des lipides du lait}

\section{1 / Acides gras saturés et mono- insaturês de structure cis}

\section{a / Acides gras à chaîne courte et moyenne}

La majeure partie des AG provenant de la lipogenèse de novo sont saturés ( $C$ 4:0 à C16:0), car la delta-9 désaturase mammaire n'a qu'une faible activité sur les AG ayant moins de 18 atomes de carbone. Toutefois, une faible proportion du C14:0 et du C16:0 sont désaturés en C14:1 cis et C16:1 cis. Les AG à longue chaîne (ayant au moins 16 atomes de carbone) sont de puissants inhibiteurs de l'acétyl-CoA carboxylase et de la lipogenèse de novo dans les cellules mammaires. Cet effet inhibiteur est d'autant plus marqué que les AG ont une longueur de chaîne plus élevée, sont plus insaturés et sont riches en doubles liaisons de structure trans.

Lorsque la disponibilité en AG à 18 atomes de carbone augmente (en raison soit d'un apport alimentaire accru, soit d'une mobilisation des lipides corporels), on assiste à une diminution de la sécrétion et surtout des teneurs en AG à chaîne moyenne (de C8:0 à C14:0 ou C16:0). Ceci est intéressant car les acides laurique, myristique et palmitique sont réputés athérogènes. Les teneurs des $A G$ à chaîne moyenne diminuent plus que leurs sécrétions, en raison de leur dilution dans une plus grande quantité d'AG à longue chaîne. Les AG alimentaires ont, en outre, un effet négatif indirect sur la lipogenèse de novo 
mammaire, en raison de la diminution du niveau d'ingestion de matière organique fermentescible et du rapport acétate : propionate dans le rumen. Ces modifications diminuent en effet la disponibilité en acétate et en $\beta$-hydroxybutyrate, qui sont des précurseurs de la lipogenèse mammaire.

L'herbe pâturée a des effets voisins de ceux de la supplémentation en AG à 18 atomes de carbone. En effet, lorsqu'on la compare à des régimes à base d'ensilage d'herbe, on observe une diminution des teneurs en acides myristique et palmitique du lait (revue de Chilliard et al 2001).

Si, par contre, les suppléments lipidiques sont riches en certains AG à chaîne moyenne, les teneurs en ces AG sont augmentées dans le lait. C'est le cas des savons de calcium d'huile de palme qui accroissent la teneur en acide palmitique $(+21 \mathrm{mg} / \mathrm{g}$ pour un apport moyen de $770 \mathrm{~g} / \mathrm{j}$ dans 6 essais). Un tel effet n'est pas favorable pour la qualité nutritionnelle du lait.

Contrairement aux AG à chaîne moyenne, les teneurs en $A G$ à courte chaîne $(C 4: 0, C 6: 0$ et C:0 dans une moindre mesure) ne sont généralement que peu ou pas diminuées par un apport accru de lipides dans la ration (tableaux 2 et 3), ou par la mobilisation des lipides corporels. Cette particularité est probablement due au fait que ces AG sont synthétisés en partie par des voies métaboliques indépendantes de l'acétyl-CoA carboxylase, et parce que l'oxydation des AG longs en corps cétoniques accroît la disponibilité en butyrate pour la glande mammaire. Ce n'est

Tableau 2. Effets de la supplémentation en suif, protégé $(P)$ ou non (NP), sur la sécrétion et la composition en acides gras du lait de vache (d'après Chilliard et al 2001).

\begin{tabular}{|c|c|c|c|c|}
\hline Régime & Témoin ${ }^{(1)}$ & Suif $N P^{(1)}$ & Témoin $^{(2)}$ & Suif $\mathrm{P}^{(2)}$ \\
\hline \multicolumn{5}{|l|}{ Sécrétion des acides gras $(\mathrm{g} / \mathrm{j})$} \\
\hline C4 & 27 & 29 & 29 & $41^{*}$ \\
\hline $\mathrm{C} 6+\mathrm{C} 8$ & 34 & $25^{*}$ & 31 & $26^{* *}$ \\
\hline $\mathrm{C} 10+\mathrm{C} 12$ & 65 & $33^{* *}$ & 69 & $38^{* *}$ \\
\hline C14 & 109 & $79^{* *}$ & 114 & $86^{\star *}$ \\
\hline C16 & 285 & 267 & 302 & 307 \\
\hline C18 & 80 & 94 & 88 & $154^{*}$ \\
\hline C18:1 & 187 & $264^{* *}$ & 191 & $329^{* *}$ \\
\hline C18:2 & 22 & $17^{*}$ & 26 & 27 \\
\hline Matière grasse sécrétée $(\mathrm{g} / \mathrm{j})$ & 909 & 913 & 903 & $1073^{*}$ \\
\hline \multicolumn{5}{|l|}{ Composition en acides gras (\% pondéral) } \\
\hline C6 à C14 & 23,6 & $15,3^{* *}$ & 23,7 & $14,0^{* *}$ \\
\hline C16 & 32,4 & 29,6 & 33,4 & $28,7^{\star}$ \\
\hline C18 & 8,5 & 10,4 & 9,7 & $14,1^{* *}$ \\
\hline C18:1 & 20,2 & $29,0^{* *}$ & 21,1 & $30,8^{* *}$ \\
\hline Index d'athérogénicité ${ }^{(3)}$ & 3,5 & 2,0 & 3,3 & 1,7 \\
\hline
\end{tabular}

(1) 6 lots de vaches recevant un régime témoin ou un régime contenant $4,4+0,7 \%$ de la matière sèche sous forme de suif non protégé.

(2) 5 lots de vaches recevant un régime témoin ou un régime contenant $4,5+1,5 \%$ de la matière sèche sous forme de suif protégé.

(3) $\mathrm{C} 12: 0+4 \mathrm{C} 14: 0+\mathrm{C} 16: 0$ / somme des acides gras insaturés (Ulbricht et al 1991).

${ }^{*}$ ou ${ }^{* *}$ : différent du lot témoin $(P<0,05$ ou $P<0,01)$.

Tableau 3. Effets de supplémentations en huiles ou graines végétales sur la sécrétion et la composition des lipides du lait de vache (exprimés par différence entre le lot supplémenté et le lot témoin).

\begin{tabular}{|c|c|c|c|c|c|c|c|c|}
\hline \multirow{3}{*}{\begin{tabular}{|l|} 
Supplément \\
Lipides du régime $(\%)$ \\
\end{tabular}} & \multicolumn{2}{|c|}{ Lin } & \multicolumn{2}{|c|}{ Tournesol } & \multicolumn{2}{|c|}{ Soja } & \multicolumn{2}{|c|}{ Colza } \\
\hline & \multirow{2}{*}{$\begin{array}{l}\text { Huile }^{(1)} \\
+3,0\end{array}$} & $\begin{array}{l}\text { Graine } \\
\text { entière }\end{array}$ & Huile $^{(1)}$ & $\begin{array}{l}\text { Graine } \\
\text { aplatie }^{(3)}\end{array}$ & Huile ${ }^{(4)}$ & $\begin{array}{l}\text { Graine } \\
\text { broyée }^{(4)}\end{array}$ & Huile ${ }^{(5)}$ & $\begin{array}{l}\text { Graine } \\
\text { broyée }^{(6)}\end{array}$ \\
\hline & & $+4,0$ & $+3,0$ & $+4,2$ & $+4,2$ & $+3,2$ & $+4,3$ & $+5,3$ \\
\hline Matière grasse du lait (g/j) & -90 & +8 & -110 & -259 & -155 & +81 & -169 & +18 \\
\hline Taux butyreux $(\mathrm{g} / \mathrm{kg})$ & $-5,2$ & $+1,2$ & $-7,0$ & $-7,1$ & $-13,9$ & $-0,4$ & $-3,9$ & $+0,1$ \\
\hline $\begin{array}{l}\text { Acides gras du lait } \\
\text { (\% des AG totaux) }\end{array}$ & & & & & & & & \\
\hline $\mathrm{C} 4: 0$ & $-0,2$ & $+0,1$ & $-0,1$ & $-1,2$ & $+0,2$ & $+0,7$ & $-0,4$ & $+0,2$ \\
\hline C6 à C8:0 & $-0,7$ & $-0,3$ & $-0,9$ & $-2,1$ & $-1,6$ & $-0,4$ & $-0,7$ & $-0,4$ \\
\hline C10 à C14:0 & $-4,2$ & $-4,6$ & $-5,9$ & $-8,6$ & $-13,1$ & $-8,4$ & $-4,7$ & $-5,2$ \\
\hline C16:0 & $-8,9$ & $-5,2$ & $-9,7$ & $-8,7$ & $-14,7$ & $-11,5$ & $-4,7$ & $-8,9$ \\
\hline C18:0 & $+2,1$ & $+4,9$ & $+2,9$ & $+4,1$ & $+3,9$ & $+7,7$ & $+3,1$ & $+5,5$ \\
\hline C18:1 & $+8,5$ & $+5,4$ & $+11,8$ & $+15,8$ & $+25,9$ & $+13,9$ & $+8,5$ & $+10,4$ \\
\hline dont C18:1 trans & $(+5,5)$ & nd & $(+6,8)$ & $(+7,4)$ & $(+4,5)$ & $(+0,4)$ & $(+1,4)$ & nd \\
\hline C18:2 & $-0,3$ & $+0,2$ & $+0,3$ & $+1,3$ & $+0,9$ & $+1,8$ & $+0,6$ & $-0,6$ \\
\hline C18:3 & $+0,3$ & $+0,3$ & $-0,1$ & $+0,3$ & nd & $+0,1$ & 0,0 & 0,0 \\
\hline Index d'athérogénicité(7) & $-1,8$ & $-1,2$ & $-2,2$ & -2 & $-3,8$ & $-2,8$ & $-1,1$ & $-1,8$ \\
\hline
\end{tabular}

(1) A. Ferlay et Y. Chilliard (non publié), (2) Kennelly 1996, (3) Casper et al 1988, (4) Steele et al 1971, (5) Tesfa et al 1991, (6) Murphy et al 1990, (7) C12:0 + 4 C14:0 + C16:0 / somme des acides gras insaturés (Ulbricht et al 1991). nd : non déterminé 
que dans les cas de très forte inhibition de la lipogenèse mammaire (par exemple avec des rations très riches en concentrés amylacés et pauvres en fibres, ou lors de supplémentations en huiles de poisson) que les teneurs en AG à courte chaîne sont diminuées, mais toujours moins que celles des AG à chaîne moyenne. La stabilite de la teneur en acide butyrique du lait est intéressante puisque cet acide gras a des effets bénéfiques sur la santé humaine.

\section{b / Acides gras à 18 atomes de carbone}

La sécrétion d'acide stéarique dans le lait peut être accrue soit par un apport d'acide stéarique alimentaire, soit par l'apport d'AG insaturés à 18 atomes de carbone en raison de leur hydrogénation en acide stéarique dans le rumen. II en est de même pour l'acide oléique, en raison soit de sa sécrétion directe, soit de sa synthèse par action de la désaturase mammaire sur l'acide stéarique.

II est souhaitable d'accroître le rapport C18:1 cis / C18:0 pour diminuer la dureté des beurres, et pour améliorer leur qualité nutritionnelle, notamment pour limiter le risque athérogène chez l'homme. Ce rapport est régulé à la fois par les disponibilités respectives en ces deux AG, par l'activité de la désaturase mammaire et par les facteurs qui modulent cette activité (disponibilité en AGPI, notamment).

Parmi les facteurs alimentaires modifiant la sécrétion des acides stéarique et oléique du lait, la supplémentation en suif (riche en C16:0, C18:0 et C18:1 ci s) a été largement étudiée. Dans le cas du suif non protégé, on assiste à une augmentation (linéaire jusqu'à $5 \%$ de suif ajouté dans la ration) de la sécrétion d'acide oléique, compensée par une diminution des sécrétions d'AG de $\mathrm{C} 6$ à $\mathrm{C} 14$, si bien que la sécrétion de matières grasses ne varie pas (tableau 2). Avec du suif protégé, apporté en quantité équivalente, on observe le même effet dépressif sur les AG de C6 à C14, mais la sécrétion d'acide stéarique augmente et celle d'acide oléique augmente spectaculairement, ce qui entraîne une forte augmentation de la sécrétion de matières grasses (ces réponses sont linéaires jusqu'à environ 7,5\% de suif protégé dans la ration). L'acide palmitique varie peu car son apport exogène compense la diminution de sa synthèse.

D'un point de vue mécanistique, on peut donc penser que le suif non protégé modifie, par rapport au suif protégé, le métabolisme ruminal et/ou mammaire d'une manière qui limite significativement l'augmentation de la sécrétion des acides stéarique et oléique, bien qu'il n'accroisse que faiblement la production d'AG trans dans le rumen. Dans le cas du suif protégé, les effets sur la composition en AG du lait sont très voisins de ceux qui résultent de la mobilisation des lipides corporels lorsque les vaches sont sous-alimentées. D'un point de vue pratique, il apparaît que les suifs modifient favorablement la qualité nutritionnelle des AG du lait, en abaissant nettement leur index d'athérogénicité (tableau 2). Toutefois, ces suppléments sont maintenant interdits en Europe, en application du principe de précaution eu égard aux risques putatifs de contamination par des prions.

Parmi les autres moyens d'accroître la sécrétion d'acide oléique du lait, on peut citer la distribution d'oléamide (J enkins 1998) ou d'huiles ou graines végétales riches en acide oléique (tournesol oléique, colza) et efficacement protégées. Une exception est la graine de coton protégée, qui diminue fortement le rapport C18:1 / C18:0 (Gulati et al 1997), car elle contient des inhibiteurs de la désaturase mammaire. Lorsqu'on distribue des huiles végétales non protégées, riches en acides oléique, linoléique ou linolénique, on accroît le flux absorbé d'acide stéarique produit dans le rumen, puis sa transformation en acide oléique dans la mamelle, si bien que les proportions de ces deux AG sont accrues dans le lait (tableau 3). Toutefois, il y a aussi un accroissement de la production de C18:1 trans dans le rumen et dans le lait.

\section{2 / Acides gras polyinsaturés}

Les AGPI ne sont pas synthétisés par les tissus des ruminants, de sorte que leur concentration dans le lait dépend étroitement des quantités absorbées dans l'intestin et donc des quantités quittant le rumen. Cette quantité peut être accrue par l'apport alimentaire d'AGPI et par les facteurs reduisant I'hydrogénation ruminale, incluant l'emprisonnement des AG dans les cellules végétales, ou l'utilisation de techniques d'encapsulation des huiles riches en AGPI.

\section{a / Acide linoléique}

Avec la plupart des rations non supplémentées en lipides, la proportion d'acide linoléique dans les AG du lait est généralement comprise entre 2 et $3 \%$ Lorsque les rations sont enrichies en graines ou huiles riches en acide linoléique comme celles de soja ou de tournesol, ce pourcentage ne dépasse pas 3 à $4 \%$, l'accroissement par rapport au régime témoin étant rarement supérieur à 1,5\% (tableau 3). Ce n'est qu'exceptionnellement qu'une proportion de $7 \%$ d'acide linoléique dans les AG du lait a été observée (Chouinard et al 1997). Il est donc clair que I'hydrogénation poussée de l'acide linoléique dans le rumen limite fortement son incorporation dans les AG du lait.

Il est souvent dit qu'un moyen de limiter I'hydrogénation ruminale est la distribution des lipides sous forme de graine plutôt que d'huile, car l'enveloppe des graines limiterait l'accessibilité des lipides aux bactéries. Par ailleurs, dans le cas du soja, elle tend à limiter la diminution du rapport acétate / propionate dans les acides gras volatils du rumen (Jouany et al 2000), diminution souvent engendrée par les suppléments de lipides insaturés. Aussi, la supplémentation en graine de soja ne diminue pas le taux butyreux et accroît plus fortement que l'huile la proportion d'acide linoléique dans les matieres grasses du lait (tableau 3). II semble toutefois que les enveloppes du tournesol et du colza 
aient un effet protecteur moindre (tableau 3) et que celle du coton ait un effet intermédiaire. Des recherches supplémentaires sont nécessaires pour confirmer ces tendances car il n'existe que peu de comparaisons directes entre huile et graine. Par ailleurs, l'extrusion de la graine de soja décroît légèrement la proportion d'acide linoléique (Chouinard et al 1997), probablement en raison d'une libération plus rapide de l'huile des structures végétales, qui faciliterait leur hydrogénation.

Les suppléments lipidiques peuvent être protégés de la dégradation dans le rumen. J usqu'à présent, le seul moyen de protection efficace est l'encapsulation des lipides par une coque de protéines tannées. Cette technique a permis, dans des conditions expérimentales, d'obtenir des laits dont la matière grasse contenait jusqu'à $35 \%$ d'acide linoléique. Dans des conditions plus proches de la pratique, des proportions de 15 à $20 \%$ ont été atteintes avec des suppléments d'huiles de soja, colza, coton, carthame ou tournesol encapsulées (McDonald et Scott 1977). La limite de cette pratique alimentaire tient au coût des procédés de fabrication et à l'utilisation du formaldéhyde, dont l'emploi est parfois mis en question. D'autres techniques de protection des lipides, comme la présentation sous forme de savons, n'empêchent pas I'hydrogénation des lipides polyinsaturés (Ferlay et al 1993), car les savons se dissocient dans le rumen lorsque le $\mathrm{pH}$ diminue. En conséquence, la teneur en acide linoléique du lait n'est pas accrue (Ferlay et al 1992, Enjalbert et al 1997). La distribution d'amides d'AG, au stade expérimental aux Etats-Unis, augmente de 2 points la teneur en acide linoléique des AG du lait, suggérant une protection faible (J enkins et al 1996).

Figure 3. Teneurs en acides linoléique et linolénique du lait de vaches recevant des rations riches en différents fourrages (d'après Chilliard et al 2001).

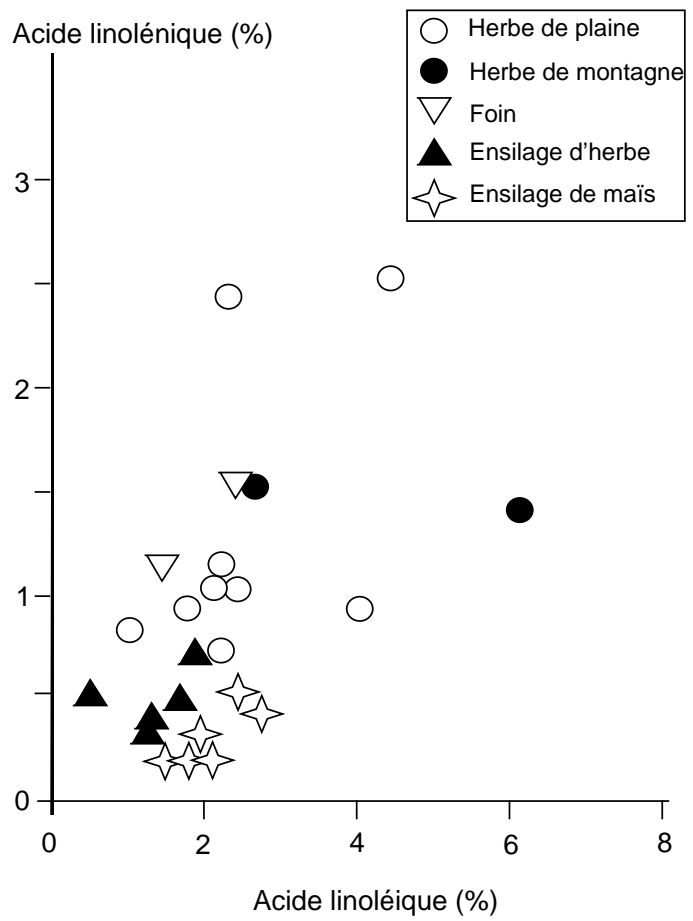

Parmi les sources potentielles permettant d'accroître l'acide linoléique, la graine de soja semble la plus prometteuse. II faut toutefois tenir compte d'une valeur azotée plus faible que celle du tourteau de soja, du fait de la forte dégradabilité des protéines de la graine. Par ailleurs, l'accroissement de la proportion d'acide linoléique dans les produits laitiers et la viande ne constitue pas en soi un objectif, dans la mesure où l'amélioration de la valeur nutritionnelle de ces produits passe par un accroissement du rapport linolénique / linoléique. La supplémentation en soja reste toutefois le moyen le plus efficace, parmi les lipides végétaux non protégés, pour diminuer l'index d'athérogénicité des matières grasses laitières (tableau 3).

\section{b / Acide linolénique}

L'herbe verte est la principale source d'acide linolénique, ce qui explique que les laits provenant de rations à base d'herbe soient plus riches en C18:3 que ceux de rations à base de maïs (figure 3 ). Toutefois, le fanage réduit considérablement la concentration de l'acide linolénique dans les fourrages, en raison de diminutions simultanées de la teneur en $A G$ et de la proportion d'acide linolénique, alors que les modifications dues à l'ensilage sont plus modérées. C'est principalement au printemps et à l'automne que la teneur en AG de I'herbe est la plus élevée. Cela explique que la mise à I'herbe entraîne un fort accroissement de la teneur en acide linolénique du lait (figure 4), qui peut atteindre 2,5\% des AG totaux (Decaen et Ghadaki 1970). En revanche, des teneurs ne dépassant pas $1 \%$ ont été observées au pâturage (Lawless et al 1998), probablement en raison d'un stade de végétation plus avancé.

Figure 4. Effets de la mise à l'herbe sur la teneur du lait de vache en diènes (principalement C18:2), diènes conjugués (principalement CLA) et triènes (principalement C18:3) (d'après Kuzdzal-Savoie et Kuzdzal 1961).

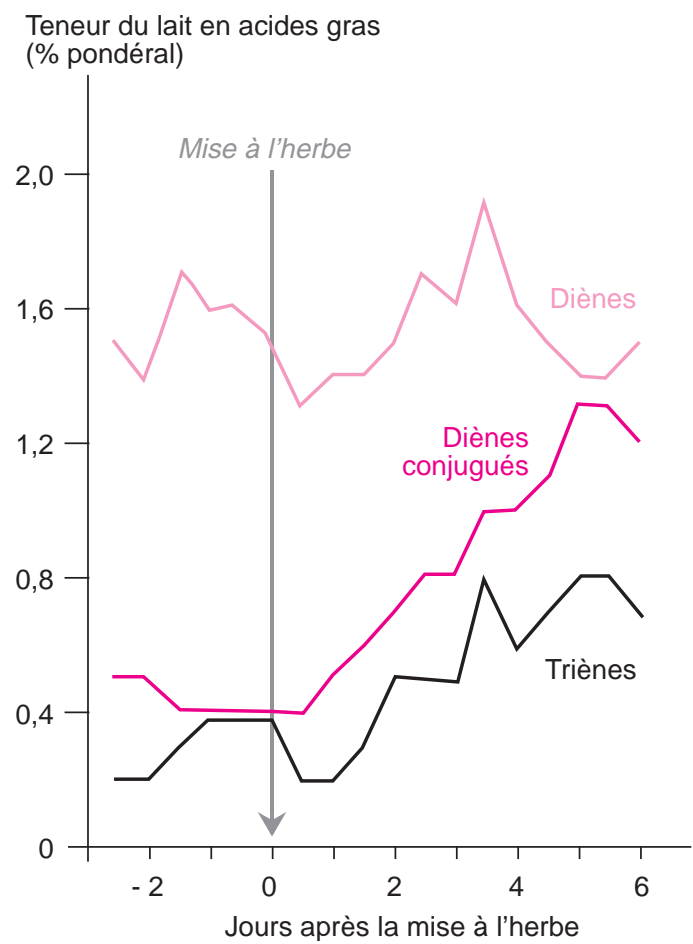

INRA Productions Ani males, décembre 2001 
Mis à part les fourrages, seule la graine de lin fournit des apports substantiels en acide linolénique, qui représente plus de $50 \%$ des AG. II faut toutefois avoir à l'esprit qu'une vache consommant $20 \mathrm{~kg}$ de MS par jour ingère quotidiennement de 300 à $400 \mathrm{~g}$ d'acide linolénique sur pâturage de printemps ou d'automne, et la même quantité si elle reçoit une ration hivernale additionnée de 3 à $4 \%$ d'huile de lin.

Peu d'essais ont été réalisés en supplémentant la ration des vaches en graine ou en huile de lin. Kennelly (1996) a observé un accroissement de la teneur en acide linolénique du lait de $+0,6 \%$ des AG totaux. D'autres auteurs n'ont pas trouvé d'accroissement (Kelly et al 1998), ou des accroissements de l'ordre de $+0,3 \%$ (Brunschwig et al 1996 et 1997, A. Ferlay et Y. Chilliard, non publié ; tableau 3). La variabilité des résultats obtenus rend nécessaire l'obtention de nouvelles références, en distinguant en particulier l'effet de la graine de celui de I'huile. Les résultats du tableau 3 suggèrent toutefois que la graine de lin n'augmente pas plus que l'huile la teneur en C18:3 du lait.

Comme avec les suppléments d'huiles riche en acide linoléique, la protection de l'huile de lin par encapsulation est le seul moyen de garantir une teneur élevée en acide linolénique dans le lait. Un régime extrême comportant $20 \%$ d'huile de lin encapsulée par des protéines tannées a permis d'atteindre $20 \%$ d'acide linolénique dans les AG totaux du lait (McDonald et Scott 1977). Avec un supplément plus raisonnable de $410 \mathrm{~g}$ d'huile de lin protégée par jour, une proportion de 6,4\% d'acide linolénique a été atteinte (Goodridge et Ingalls 1998). Le traitement au formaldéhyde de la graine de lin permet d'accroître plus fortement la teneur en C18:3 du lait de chevre que la graine non tannée ( +11 vs $+6 \mathrm{mg} / \mathrm{g}$; Chilliard et Lamberet 2001 et non publié).

Un facteur essentiel qui peut limiter I'utilisation de la graine de lin dans la ration des vaches laitières est son effet fortement négatif sur la digestion des parois végétales, s'accompagnant d'une chute très importante du nombre de protozoaires et d'une orientation du profil des AGV vers le propionate (J ouany et al 2000). En fait, toutes les données de digestion ont été obtenues sur des moutons à l'entretien recevant de l'huile de lin. Une confirmation est nécessaire sur bovins en production recevant de la graine ou de l'huile de lin. Les résultats zootechniques laissent en effet penser que l'apport de matières grasses de lin aux bovins n'entraîne pas de diminution notable de la valeur énergétique des rations, et donc de leur digestibilité.

Un autre facteur susceptible de freiner l'utilisation de la graine de lin est la difficulté d'approvisionnement qui pourrait se produire en cas de développement de cet aliment, car les surfaces cultivées en France sont limitées. Par ailleurs, des études sont nécessaires pour analyser l'effet de traitements technologiques comme l'extrusion sur la vitesse d'hydrogénation des lipides, mais aussi sur la destruction des composés cyanogènes présents dans la graine native.
La graine de colza contient quant à elle une quantité notable d'acide linolénique, dont une partie est susceptible d'être sécrétée dans le lait. Toutefois, comme déjà noté pour le C18:2, l'apport de graine ou d'huile de colza ne permet pas d'accroître le C18:3 du lait (tableau 3).

\section{c / E PA (eicosapentanoic acid) et DHA (docosahexanoic acid)}

La sécrétion des $A G$ de la série $n-3$ à 20 et 22 atomes de carbone (C20:5 ou EPA, et C22:6 ou DHA, respectivement) peut être accrue lorsqu'on apporte des huiles marines (de poissons ou d'algues) dans la ration des vaches (revue de Chilliard et al 2001). L'efficacité du transfert de la ration au lait est cependant faible $(2,6 \%$ pour l'EPA et $4,1 \%$ pour le DHA) en raison de fortes hydrogénations ruminales, en particulier pour I'EPA. Des efficacités de transfert supérieures, comprises entre 16 et $33 \%$, sont observées lors d'infusions post-ruminales d'huile de poisson. L'accroissement de la teneur des AG du lait en EPA + DHA est donc faible lorsqu'on ajoute de l'huile de poisson dans la ration des vaches, et ne dépasse que rarement $0,5 \%$ des AG totaux. En outre, l'efficacité du transfert du DHA diminue au cours du temps (entre la deuxième et la sixième semaine de distribution d'algues), suggérant que la microflore du rumen s'adapte progressivement à la présence de ces huiles (Franklin et al 1999). Les huiles marines dites protégées ont souvent des effets sur la composition en $A G$ du lait comparables à ceux des huiles non protégées (revue de Chilliard et al 2001). Il est donc probable qu'une protection incomplète ne suffit pas à empêcher les effets très puissants de ces huiles.

La chute de la sécrétion lipidique mammaire est mieux expliquée par l'ingestion d'EPA (figure 5) que par celle de DHA, ou par la somme EPA + DHA. On peut donc faire l'hypothèse que l'hydrogénation ruminale de I'E PA produirait spécifiquement des AG ayant des doubles liaisons de structure trans, plus fortement inhibiteurs de la lipogenèse mammaire. Toutefois les algues marines, pauvres en EPA, ont aussi un fort effet inhibiteur sur la lipogenèse mammaire. En outre, les faibles quantités d'EPA échappant à l'hydrogénation ruminale pourraient exercer un effet inhibiteur direct sur l'activité de la delta-9 désaturase mammaire.

\section{3 / Acides gras trans et acide linoléique conjugué}

\section{a / E ffets sur la lipogenèse ma mmaire}

La supplémentation en huiles marines à faibles doses entraîne une forte chute du taux butyreux (cf. tableau 1) et de la sécrétion des lipides dans le lait (figure 5). Ceci pourrait être lié à la production d'AG trans dans le rumen et à leurs effets négatifs sur la lipogenèse mammaire (figure 5). L'augmentation de la teneur en C18:1 trans du lait est due majoritairement (74-82 \%) à l'acide trans-vaccénique. 
Figure 5. Relations entre l'ingestion d'EPA( C20:5, n-3) ou la réponse du C18:1 trans du lait et la réponse de la sécrétion de matières grasses du lait chez des vaches recevant des huiles marines et différents types de fourrages (d'après Chilliard et al 2001).

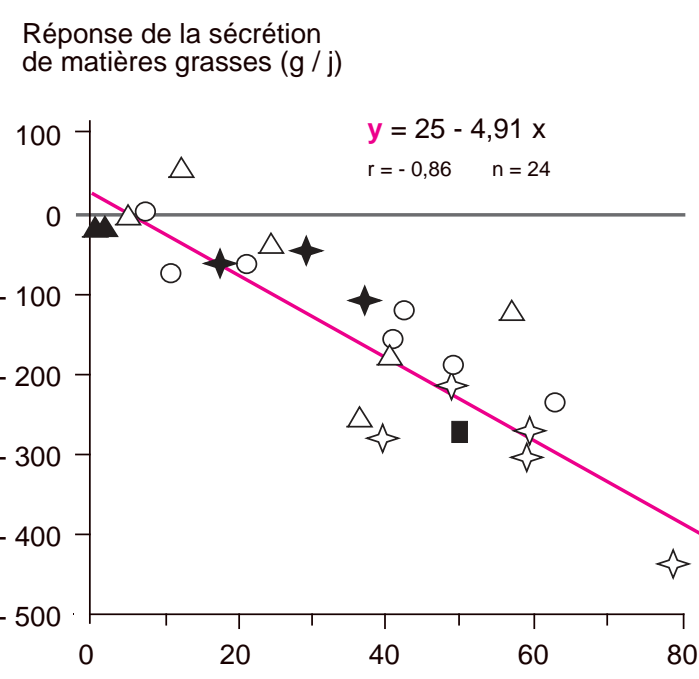

Ingestion d'EPA d'huile de poisson ( $\mathrm{g} / \mathrm{j}$ )
Réponse de la sécrétion de matières grasses $(g / j)$

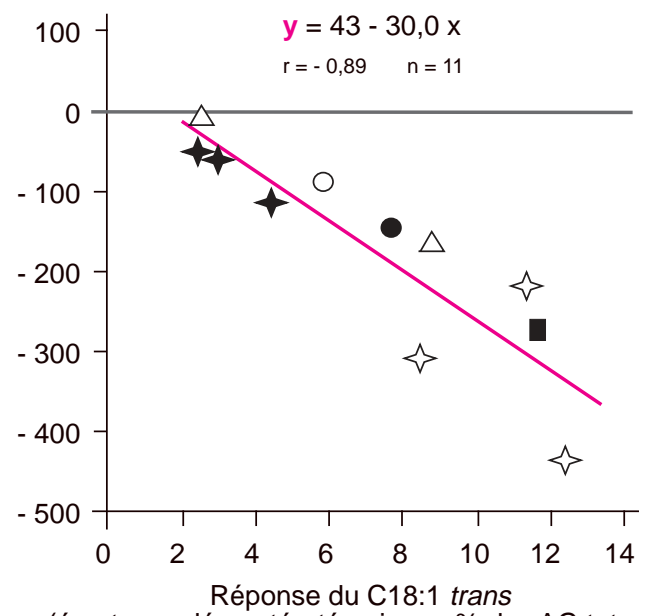

(écart supplémenté - témoin, en \% des AG totaux)

$$
\begin{aligned}
& \triangle \text { Foin } \\
& \triangle \text { Ensilage d'herbe }
\end{aligned}
$$

Le taux butyreux du lait est, par ailleurs, fortement diminué par les régimes pauvres en fibres et riches en céréales, et/ou par l'administration d'huiles végétales insaturées et non protégées. L'implication des C18:1 trans dans cette diminution a été suggérée depuis longtemps (Davis et Brown 1970). Les résultats du tableau 3 montrent par ailleurs que les graines de lin et de soja accroissent plus fortement la teneur en acide stéarique du lait, et moins fortement celle en C18:1 trans, lorsqu'on les compare aux huiles correspondantes (la différence étant moins nette dans le cas des graines de tournesol et de colza). II semble donc que l'hydrogénation soit plus complète (bien que concernant un pourcentage un peu plus faible des AGPI apportés) lorsque les lipides sont apportés dans les graines, probablement parce qu'une libération progressive perturbe moins l'écosystème ruminal qu'un apport d'huile en 2 repas par jour (voir aussi Morales et al 2000). Un effet comparable est observé lorsque l'apport d'huile est fractionné en 24 repas par jour (Banks et al 1980).

Néanmoins, dans certaines études, une augmentation de la proportion du C18:1 trans total dans le lait n'est pas accompagnée d'une chute du taux butyreux. Ceci a été précisé récemment par Piperova et al (2000) et Bauman et Griinari (2001), montrant que des régimes pauvres en fibres et supplémentés en huile de soja augmentent fortement les proportions du C18:1 10-trans (et non celle du C18:1 11-trans), mais aussi du C18:2 10-trans, 12-ci s et du C18:2 7-trans 9-cis dans le lait. Ces régimes induisent une baisse des activités de I'ACC et de I'AGS, ainsi qu'une diminution de l'expression du gène de l'ACC. II est possible que le CLA 10-trans, 12-cis résulte de modifications des biohydrogénations ruminales et

\footnotetext{
Foin + ensilage de maïs

- Ensilage d'herbe + foin

Ensilage de maïs + ensilage d'herbe

Haylage de luzerne + ensilage de maïs
}

soit un des précurseurs du C18:1 10-trans formé dans le rumen. De plus, une infusion duodénale d'un mélange de C18:1 trans diminue fortement le taux butyreux, mais ne diminue pas la concentration du C18:1 9-cis dans le lait, suggérant que la baisse du taux butyreux ne serait pas due principalement à une inhibition de la delta-9 désaturase (Romo et al 2000).

Différents isomères du CLA (en particulier, les 9-cis, 11-trans et 10-trans, 12-cis) ont été infusés dans le duodénum de vaches laitières durant des périodes de 3 à 5 jours. Les taux de transfert du duodénum vers le lait des différents isomères du CLA sont différents : de l'ordre de 20 à $30 \%$ pour les isomères 9-cis, 11-trans ; 8-cis 10-trans et 11-ci s,13-trans et de l'ordre de 10 à $20 \%$ pour le 10-trans, 12-ci s (Chouinard et al 1999). L'isomère 10-trans, 12-cis induit de fortes diminutions du taux butyreux (de 25 à $52 \%$ avec des doses croissantes d'isomère) et des proportions d'AG à chaînes courtes et moyennes ( 25 à 50 \%) (Loor et Herbein 1998, Baumgard et al 2000 et 2001). De même, il apparaît qu'un mélange d'isomères contenant en quantités égales du 8-cis, 10-trans et du 9-cis, 11-trans diminue aussi la synthèse de novo des AG (Chouinard et al 1999). Ainsi, les isomères du CLA portant la double liaison sur le carbone 10 pourraient inhiber la lipogenèse. Les mécanismes impliqués dans cette inhibition seraient une réduction de l'expression et des activités des enzymes ACC et AGS par le 10-trans, 12-cis (et non par le 9-cis, 11-trans), comme montré dans des cultures de cellules épithéliales mammaires (Matitashvili et Bauman 2000). Une diminution de la sécrétion des AG à chaîne longue ( $>$ C16) lors de l'infusion du 10trans, 12-cis a aussi été observée, suggérant une réduction du prélèvement des $A G$ préformés par la lipoprotéine lipase (Baumgard et INRA Productions Ani males, décembre 2001 
al 2001). Parallèlement, le rapport C18:1/C 18:0 diminue, ce qui suggère une diminution de l'activité de la delta-9 désaturase (Chouinard et al 1999, Baumgard et al 2000 et 2001).

Ces nouveaux résultats ont conduit à réactiver la théorie de la biohydrogénation des AG comme mécanisme central expliquant les chutes de taux butyreux avec certains régimes (Bauman et Griinari 2001). Cependant, une supplémentation de la ration avec de l'huile de poisson diminue fortement le taux butyreux, sans augmentation notable du C18:2 10-trans, 12-cis, tandis que I'élévation de la teneur en acide ruménique explique 96\% de la forte augmentation des CLA (Chilliard et al 1999). Le CLA 10-trans 12-cis n'est donc pas le seul facteur susceptible de faire chuter le taux butyreux du lait. D'autres isomères du C18:2 ou du C18:3, produits dans le rumen et ayant une double liaison 10-trans, pourraient être impliqués (Bauman et Griinari, 2001 ; Bauman et al 2001). De nombreuses incertitudes demeurent encore sur les effets respectifs des différents isomères du C18:1 trans et du CLA sur les étapes successives de la synthèse des lipides tissulaires et du lait (synthèse, prélèvement, désaturation, estérification, synthèse et sécrétion des globules gras), aussi bien chez les ruminants que chez les monogastriques.

\section{b / F acteurs alimentaires de variation}

Les facteurs alimentaires influençant la composition des laits en CLA et en C18:1 trans se répartissent en 3 catégories : 1) les régimes apportant des précurseurs lipidiques (C18:2 ou C18:3) pour la formation de CLA et de C18:1 trans dans le rumen, 2) les régimes modifiant l'activité microbienne associée à I'hydrogénation ruminale des AG polyinsaturés (tableau 4) et 3) les suppléments alimentaires de CLA ou de C18:1 trans. Les combinaisons entre les différents facteurs de ces 3 catégories peuvent permettre d'induire de très larges variations des teneurs du lait en CLA et/ou en C18:1 trans.

Très peu de données relatives à l'effet d'un apport de suif dans la ration sur les teneurs du lait en C18:1 trans et en CLA sont disponibles. Les concentrations restent faibles avec un apport de 4-5\% de suif dans la ration, probablement en raison du faible taux d'AGPI dans le suif (respectivement, 0,9 à $1,6 \%$ et 0,2 à 0,5\% pour les proportions de C18:1 trans et de CLA dans les AG du lait). Toutefois, des valeurs plus élevées ont été observées avec des régimes riches en maïs et en suif (Morales et al 2000), mais sans données comparables sur un régime témoin sans suif.

Les huiles végétales riches en C18:2 (tournesol, soja) augmentent fortement la teneur en CLA du lait. Cet effet est linéaire lors d'addition de quantités croissantes d'huile de soja à la ration (jusqu'à $4 \%$ de la $M S$, au moins) (Chilliard et al 2000). Parallèlement à des effets directs sur la production de CLA et de C18:1 trans, l'acide linoléique inhibe probablement la réduction finale des C18:1 trans, et augmente par conséquent leur accumulation dans le rumen. L'addition de savons de calcium de colza dans la ration augmente la teneur en CLA du lait. Ceci confirme que les AGPI des savons de calcium sont partiellement hydrogénés. De manière générale, les huiles végétales élèvent plus le taux de CLA dans le lait que les graines extrudées, ellesmêmes plus fortement que les graines crues. Ceci reflète un effet d'autant plus important que les AGPI perturbent plus largement le métabolisme ruminal, en accord avec les effets respectifs des huiles et des graines sur l'acide stéarique et le C18:1 trans du lait (tableau 3 et Bayourthe et al 2000). L'huile de lin (riche en C18:3) augmente presque autant la teneur en CLA que l'huile de tournesol (riche en C18:2). Cet effet s'expliquerait par une forte augmentation de la production de C18:1 11-trans dans le rumen (tableau 3), qui serait ensuite prélevé par la mamelle, et désaturé en acide ruménique par la delta-9 désaturase.

Pour la même quantité d'huile distribuée dans la ration, les huiles de poisson sont plus efficaces que les huiles végétales pour augmenter la teneur en CLA. Ainsi, les proportions de CLA passent de 0,2-0,6\% avec le régime témoin à 1,5-2,7 \% avec des régime supplémentés en huile de poisson (200-300 g/j ; figure 6). Il est peu probable que l'hydrogénation des AG polyinsaturés de ces huiles produise directement du CLA et du C18:1 11-trans. Néanmoins, I'huile de poisson augmente, dans le rumen et dans le lait, la concentration de C18:1 11-trans, vraisemblablement par inhibition, dans le rumen, de la réduction de cet AG.

Une relation linéaire existe entre les concentrations du CLA et du C18:1 trans dans le lait pour une grande diversité de régimes alimentaires (figure 7). Cependant, le rapport CLA/ C18:1 11-trans plafonne avec I'huile de poisson

Tableau 4. Effets des facteurs alimentaires sur la teneur en CLA du lait de vache (d'après Chilliard et al 2000 et 2001). De nombreuses interactions entre facteurs alimentaires peuvent changer les réponses multifactorielles. Il convient donc de rester prudent dans les extrapolations à partir de ce tableau.

\begin{tabular}{|c|c|c|}
\hline $\begin{array}{c}\text { Valeurs faibles } \\
(0,2-0,8 \%)\end{array}$ & $\begin{array}{l}\text { Valeurs moyennes } \\
\quad(0,8-1,6 \%)\end{array}$ & $\begin{array}{c}\text { Valeurs élevées } \\
\quad(>1,6 \%)\end{array}$ \\
\hline $\begin{array}{l}\text { Foin ou ensilage d'herbe } \\
\text { Ensilage de maïs } \\
\text { Graines de soja crues } \\
\text { Graines de coton extrudées } \\
\text { Matières grasses saturées } \\
\text { lonophores (long terme) } \\
\text { Substances tampon }\end{array}$ & $\begin{array}{l}\text { Herbe jeune } \\
\text { Régimes pauvres en fibres } \\
\text { Graines de soja chauffées ou extrudées } \\
\text { Huiles végétales (3-4 \%) } \\
\text { Savons de Ca d'huile de colza (4\%) } \\
\text { lonophores (court terme) }\end{array}$ & $\begin{array}{l}\text { Huile de tournesol ou de lin (5\%) } \\
\text { Savons de calcium d'huile de soja ou de lin (4\%) } \\
\text { Huiles marines (poissons, algues) }(2 \%)\end{array}$ \\
\hline
\end{tabular}


(figure 7). Dans ce cas, il est possible que la teneur très él evée en C18:1 11-trans dépasse la capacité de désaturation de la glande mammaire, ou que des AG spécifiques de l'huile de poisson (EPA, DHA ou des composés intermédiaires d'hydrogénation) inhibent l'activité de la delta-9 désaturase. On observe aussi un rapport CLA/C18:1 11-trans très faible dans un essai de courte durée avec des ionophores, qui augmentent très fortement le C18:1 trans (Sauer et al 1998). Cet effet est probablement

Figure 6. Relation entre l'ingestion d'huile marine (poisson ou algues) et la teneur en CLA du lait (d'après Donovan et al 2000 et Chilliard et al 2001).

CLA du lait (\% des AG totaux)

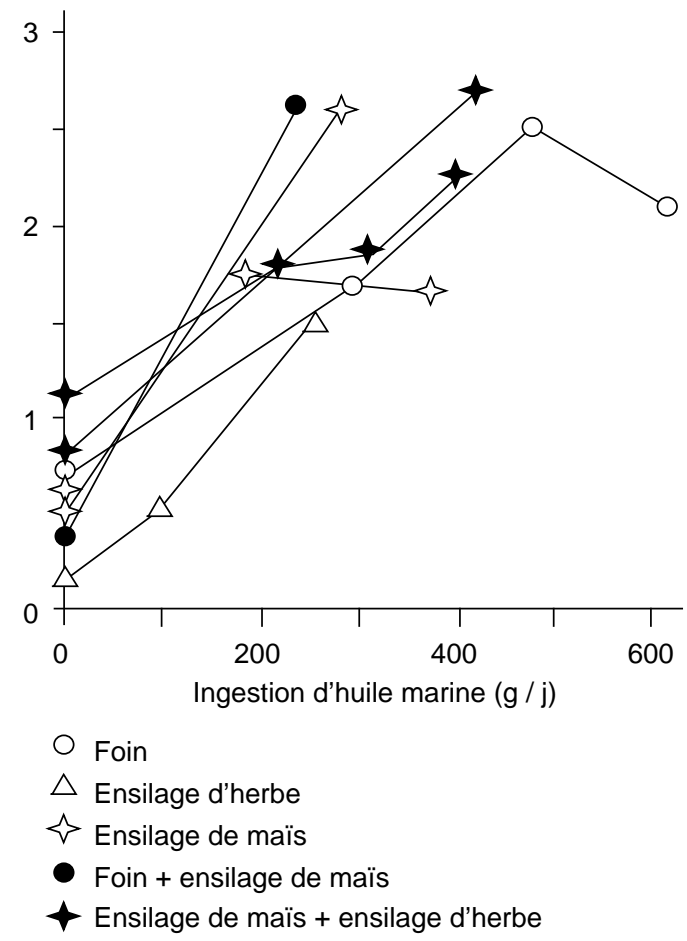

transitoire. Les effets des ionophores sont en effet très faibles dans d'autres études de plus longue durée (Griinari et Bauman 1999).

Les proportions de C18:1 trans et de CLA dans les laits obtenus à partir des régimes à base d'ensilage de maïs (plus de $60 \%$ de la ration) sont faibles (respectivement, 1,1 à 2,2\% et 0,4 à $0,6 \%$ ). La concentration du CLA dans le lait de vaches laitières passant d'un régime hivernal à de l'herbe jeune pâturée passe de 0,4$0,8 \%$ à 1,2-2,5\% (cf figure 4 et tableau 4). Néanmoins, les proportions de CLA dans le lait observées avec des animaux au pâturage (0,5 à $2,2 \%)$ sont variables. La teneur en CLA du lait augmente avec la disponibilité en herbe verte, mais varie peu avec le stade végétatif. Cependant, les concentrations du CLA dans le lait sont plus élevées au printemps et en automne qu'en été. La concentration élevée de C18:3 dans I'herbe jeune et la faible teneur de celle-ci en fibres sont 2 facteurs qui se combinent probablement pour augmenter la production de CLA et de C18:1 trans.

II n'y a que peu de données sur l'influence de l'alimentation sur les différents isomères du CLA du lait. La teneur en acide ruménique (CLA 9-cis, 11-trans) est généralement celle qui varie le plus, en raison de l'importance de sa synthèse mammaire par la delta-9 désaturase. En outre, cette enzyme synthétise du CLA 7-trans, 9-cis, qui est quantitativement le second isomère présent dans le lait. Une étude récente (Griinari et al 2000b) suggère les associations positives suivantes entre différents aliments et les isomères du CLA : pâturage et 11, 13 cis/trans; huile d'arachide et 7-trans, 9-cis; huile de tournesol et 10 trans, 12-cis; huile de lin et cis-12, trans-14 ; huile de poisson et 9-cis, 11-trans. Les rôles physiologiques respectifs de ces différents isomères et leur éventuel intérêt nutritionnel pour l'homme n'ont pas été étudiés à ce jour.

Figure 7. Relations entre les teneurs en C18:1 trans total ou en C18:1 11-trans et la teneur en CLA du lait de vache (A. Ferlay et Y. Chilliard, compilation bibliographique non publiée).

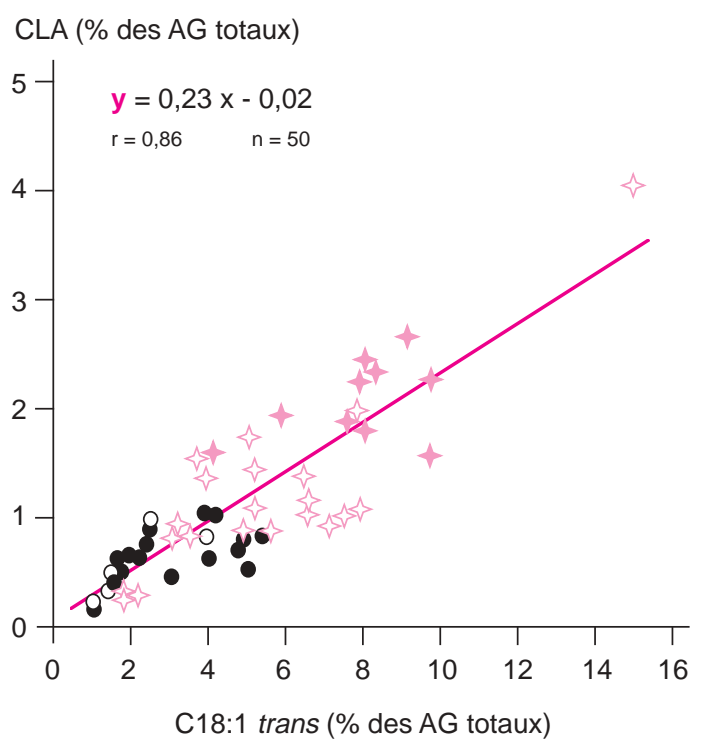

Rations non supplémentées en lipides, à base de : o herbe

- maïs
CLA (\% des AG totaux)

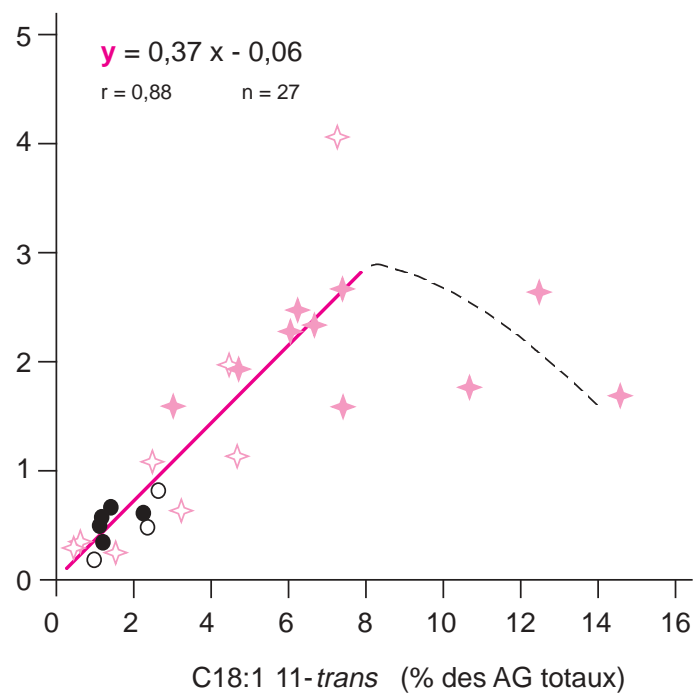

Rations supplémentées en :

$\checkmark$ matières grasses animales ou végétales

huiles marines 


\section{Conclusion}

L'alimentation permet de faire varier largement, et de façons diverses, la composition en acides gras du lait. Les progrès récents des connaissances sur les mécanismes de synthèse de ces acides gras (digestion et métabolisme), et sur leurs effets physiologiques chez l'homme, stimulent fortement les recherches en cours et leurs applications potentielles. Pour ce qui est de l'alimentation des ruminants, on cherche à mieux connaître les effets de l'utilisation des rations à base d'herbe, de nouvelles combinaisons de matières premières dans les concentrés, des traitements technologiques des graines oléagineuses, et des huiles marines. II n'existe toutefois que peu de comparaisons directes entre les grands types de rations de base (fourrages) ou les différents suppléments lipidiques (huiles, graines, traitements technologiques). Les tendances rapportées dans ce texte devront donc être confirmées.
Dans la mesure où les recommandations nutritionnelles pour l'homme peuvent encore varier dans les prochaines années, les nutritionnistes des vaches laitières doivent continuer à étudier les lois de réponses d'un grand nombre d'acides gras majeurs et mineurs, et modéliser leurs mécanismes de synthèse. Simultanément, les effets secondaires des différentes pratiques alimentaires sur la qualité sanitaire (présence éventuelle de facteurs antinutritionnels, variations de micronutriments à effet antioxydant, etc), technologique et sensorielle, et sur l'image des produits laitiers demandent à être mieux évalués. Ceci ne pourra être réalisé que dans le cadre d'approches multidisciplinaires intégrées, associant la production végétale, la technologie laitière, la génétique et la physiologie animale, la nutrition animale et humaine, et l'économie.

\section{Remerciements}

Les auteurs remercient $P$. Béraud et $Y$. Fournier pour leur assistance lors de la mise en forme du manuscrit.

\section{Références}

La plupart des références utilisées dans ce texte figurent dans les deux revues bibliographiques suivantes

Chilliard Y., Ferlay A., Mansbridge R.M., Doreau M., 2000. Ruminant milk fat plasticity: nutritional control of saturated, polyunsaturated, trans and conjugated fatty acids. Ann. Zootech. 49, 181-205.

Chilliard Y., Ferlay A., Doreau M., 2001. Effect of different types of forages, animal fat or marine oils in cow's diet on milk fat secretion and composition, especially conjugated linoleic acid (CLA) and polyunsaturated fatty acids. Livest. Prod. Sci., 70, 31-48.

\section{Références additionnelles}

Bauman D.E., Griinari J.M., 2001. Regulation and nutritional manipulation of milk fat : low-fat milk syndrome. Livest. Prod. Sci., 70, 15-29.

Bauman D.E., Corl B.A., Baumgard L.H., Griinari J .M., 2001. Conjugated linoleic acid (CLA) and the dairy cow. In: P.C. Garnsworthy and J. Wiseman (eds), Recent advances in animal nutrition, 221-250. Nottingham University Press.

Baumgard L.H., Sangster J .K., Bauman D.E., 2001. Milk fat synthesis in dairy cows is progressively reduced by increasing supplemental amounts of trans-10, cis-12 conjugated linoleic acid (CLA). J. Nutr., 131, 1764-1769.

Brunschwig Ph., Kernen P., Weill P., 1997. Effets de l'apport d'un concentré enrichi en acides gras polyinsaturés sur les performances de vaches laitières à l'ensilage de maïs. Renc. Rech. Ruminants, 4, 361.

Casper D.P., Schingoethe D.J., Middaugh R.P., Baer R.J., 1988. Lactational responses of dairy cows to diets containing regular and high oleic acid sunflower seeds. J. Dairy Sci., 71, 1267-1274.

Chilliard Y., Lamberet G., 2001. Biochemical characteristics of goat milk lipids and lipolytic system. A comparison with cow's and human milk. Effects of lipid supplementation. In: G. Freund (ed), Recent advances on goat milk quality, raw material for cheese making, 71-114. ITPLC Ed., ISBN 2-9514868-1-2.
Chouinard P.Y., Lévesque J., Girard V., Brisson G.J ., 1997. Dietary soybeans extruded at different temperatures: milk composition and in situ fatty acid reactions. J. Dairy Sci., 80, 2913-2924.

Donovan D.C., Schingoethe D.J., Baer R.J., Ryali J., Hippen A.R., Franklin S.T., 2000. Influence of dietary fish oil on conjugated linoleic acid and other fatty acids in milk fat from lactating dairy cows. J. Dairy Sci., 83, 26202628.

Griinari J.M., Corl B.A., Lacy S.H., Chouinard P.Y., Nurmela K.V.V., Bauman D.E., 2000a. Conjugated linoleic acid is synthesized endogenously in lactating dairy cows by delta 9-desaturase. J. Nutr., 130, 2285-2291.

Griinari, J.M., Bauman, D.E., Chilliard, Y., Peräjoki, P. Nurmela, K., 2000b. Dietary influences on conjugated linoleic acids (CLA) in bovine milk fat. Abstracts. 3rd Meeting of the European Section of Am. Oil Chem. Soc., 18-21th J une, Helsinki, p. 87

Murphy J .J ., Mc Neill G.P., Connolly J.F., Gleeson P.A. 1990. Effect on cow performance and milk fat composition of including full fat soyabeans and rapeseeds in the concentrate mixture for lactating dairy cows. J. Dairy Res., 57, 295-306.

Piperova L.S., Teter B.B., Bruckental I., Sampugna I. Mills S.E., Yurawecz M.P., Fritsche J ., Ku K., Erdman R.A., 2000. Mammary lipogenic enzyme activity, trans fatty acids and conjugated linoleic acids are altered in lactating dairy cows fed a milk fat-depressing diet. J. Nutr. $130,2568-2574$

Romo G.A., Erdman R.A., Terer B.B., Sampugna J., Casper D.P., 2000. Milk composition and apparent digestibilities of dietary fatty acids in lactating dairy cows abomasally infused with cis or trans fatty acids. J. Dairy Sci., 83, 2609-2619.

Steele W., Noble R.C., Moore J.H., 1971. The effects of dietary soybean oil on milk-fat composition in the cow. J. Dairy Res., 38, 49-56.

Ulbricht T.L.V., Southgate D.A.T., 1991. Coronary heart disease : seven dietary factors. Lancet, 338, 985992. 


\section{Abstract}

Dietary control of milk fat nutritional quality in the dairy cow: trans, and polyunsaturated fatty acids, and conjugated linoleic acid.

After a survey of metabolic pathways and nutrient fluxes involved in mammary lipogenesis, this review summarises the known effects of diet on ruminant milk fatty acid (FA) composition, and particularly polyunsaturated FA, trans monounsaturated FA and conjugated linoleic acid (CLA). The main dietary factors which were studied are the nature of forages (including pasture), and the supplementation of dairy rations with protected or unprotected tallow, and protected or unprotected vegetable or marine oils (fish or algae). Tallow supplementation increases the secretion of oleic acid. Milk fat percentage of linoleic acid varies from 2 to $3 \%$ in basal diets. It is increased by only 1.5 point when the diet is supplemented with oils or seeds rich in C18:2, because of an extensive ruminal biohydrogenation. Milk fat from grass diets (rich in C18:3) is higher ( 0.5 to 1 point) in linolenic acid than milk fat from maize silage diets. Linseed increases the milk fat percentage of linolenic acid by 0.3 point. Milk fat percentage of C 20:5 (E PA) and C 22:6 (DHA) is enhanced up to $0.5 \%$ of total FA by the supply in the diet of marine oils. Milk fat content can be greatly decreased by low fibre, high grain diets and/or by feeding unprotected marine oils or vegetable oils rich in unsaturated FA. A role of trans-C 18:1 isomers, including trans-11 and trans-10 C 18:1, but also of trans 10 , cis 12 CLA, in the inhibition of mammary gland lipogenesis is suggested. Diets which increase milk trans C18:1 or CLA percentage in milk fat are those which provide lipid substrates for the formation of CLA or trans C18:1 in the rumen, or those which change the microbial activity associated with ruminal biohydrogenation of polyunsaturated FA, or those which change the mammary desaturase activity. The influence of dietary factors on the different C18:1 and CLA isomers in milk fat is not well known.

CHILLIARD Y., FERLAY A., DOREAU M., 2001. Contrôle de la qualité nutritionnelle des matières grasses du lait par l'alimentation des vaches laitières : acides gras trans, polyinsaturés, acide linoléique conjugué. INRA Prod. Anim., 14, 323-335. 
